\title{
A RANDOM SIGNAL GENERATOR BY USING ANALOG ELECTRONICS
}

\author{
Leonardo Acho \\ Department of Mathematics \\ Polytechnic University of Catalunya (UPC) \\ Campus Terrassa \\ Spain \\ email:leonardo.acho@upc.edu
}

Article history:

Received 21.12.2020, Accepted 17.06.2021

\begin{abstract}
The describing function theory is a powerful mathematical tool to predict oscillations in non-linear dynamical systems. This theory is here invoked to design a random signal generator and realized by using analog electronic elements. Then, and according to experimental results, histograms of the resultant signal are shown along with the generated signal in the time domain. Finally, the proposed electronic circuit is simple and cheap to construct.
\end{abstract}

\section{Key words}

signal generator, electronics, random behaviour

\section{Introduction}

A random signal generator can be interpreted as an oscillator device but producing a random signal instead of a periodic one. This includes the chaotic oscillator systems. [Buchovecká et al., 2017; Vazquez-Medina et al., 2009]. Moreover, many chaotic oscillator circuits have been recently reported [Minati et al., 2020; Zhou et al., 2015; Piper and Sprott, 2010; Karakaya et al., 2019]. The design of these systems is based on the theory of chaotic dynamical models. Furthermore, some chaotic oscillators are too complex to implement because some of them use analog signal multipliers. [Yildirim and Kacar, 2020; Pham et al., 2017]. In contrast, there is a simple chaotic circuit but required to use of inductors. [Wu, 1987].

On the other hand, the describing function theory to predict oscillations in a dynamical system [Slotine et al., 1991] can also offer an option to design random signal generators. Hence, the main objective of this paper is to propose a novel random generator circuit by using the describing function framework. Besides, the resultant circuit is simple and easy to construct. According to experimental results, this circuit demonstrates the desired behavior. Experimental signals and histograms are shown to support our main contribution.

The rest of the paper is organized as follows. Section 2 introduces the basics of describing function theory to predict an oscillation behavior in a nonlinear dynamical system. Then, a simple analog electronic circuit to produce a random signal is conceived and shown in Section 3. Experimental results are also evidenced. Finally, in Section 4, the final remarks are written.

\section{Describing Function}

As was previously mentioned, the describing function analysis is a well-known mathematical framework to predict an oscillation behavior by a kind of nonlinear system [Slotine et al., 1991]. Basically, if a nonlinear system can be represented as in Figure 1, then an oscillating output will be observed if the following assumptions are confirmed [Slotine et al., 1991; Khalil and Grizzle, 2002; Garber and Rozenvasser, 1965; Zaitceva and Chechurin, ]:

1. The nonlinear element is a single element.

2. The nonlinear element is a time-invariant one.

3. The linear system has a limited frequency bandwidth response. 


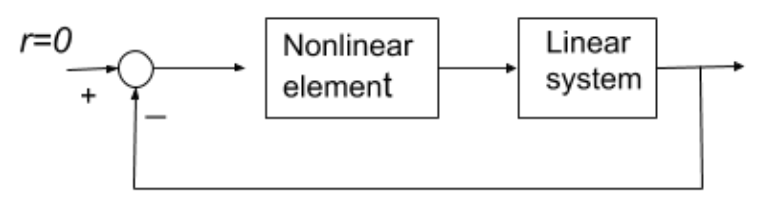

Figure 1. A nonlinear dynamical system also known as a system in the Lurie format. $r:=r(t)=0$ represents a system in which its dynamical behavior depends on itself.

In other words, an oscillating signal will be presented at the system output in Figure 1 if the nonlinear element presents saturation and the linear system is a kind of low pass filter. [Slotine et al., 1991]. For instance, operational amplifiers and transistors operated by a DC power source present saturation and have limited frequency bandwidth operation. Hence, these electronic elements satisfy some of the previous cited assumptions.

\section{A Random Signal Generator Circuit}

Based on the describing-function presentation in Section 2, we propose the circuit diagram given in Figure 2. Therefore, in this circuit, we have:

1. The given operational amplifier supplies the required negative feedback to the rest of the electronic system. Additionally, this amplifier has a finite frequency bandwidth response too.

2. The transistors have the needed saturation effect. These also produce a mixing combination of the feedback signal from the operational amplifier and are manipulated by the capacitors to these transistors.

3. The potentiometer connected to the operational amplifier is employed to tune the circuit. This potentiometer introduces a direct current component to the output signal of the operational amplifier to overcome the single polarity of the DC power supply. As a consequence of this, the output response of the circuit is linked to this parameter.

Additionally, Figure 3 shows a photo of the circuit realization for experiments.

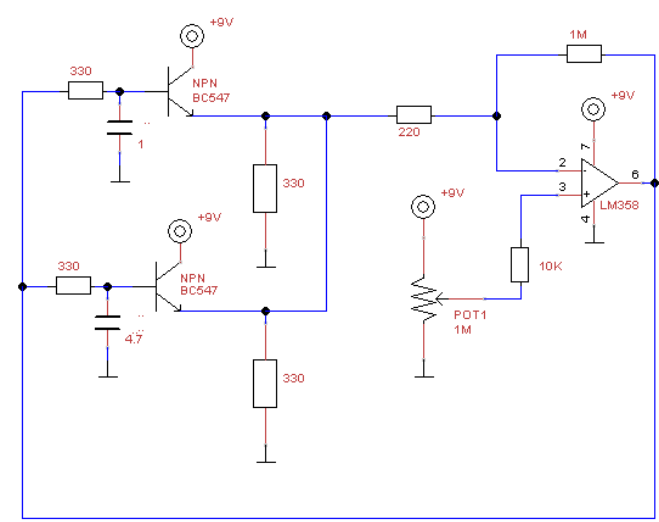

Figure 2. A random signal generator circuit. The resistances are in ohms and capacitors in microfarads. Hence, for instance, the resistance of 330 means $330 \mathrm{ohms}$, and the capacitor 1 means 1 microfarad, and so on.

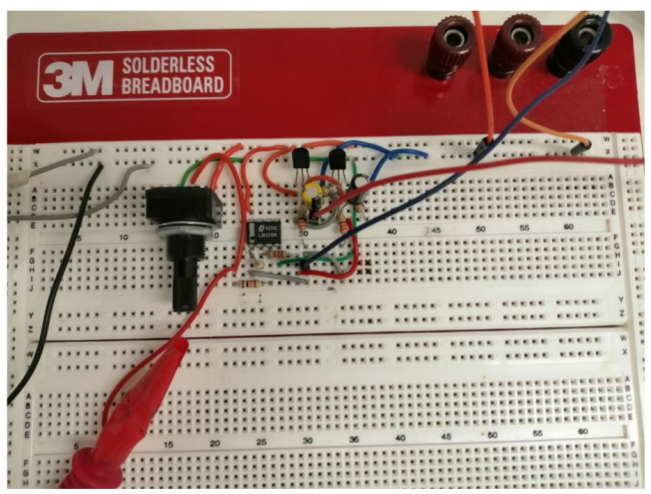

Figure 3. A photo of the schematic circuit realization.

\section{Experimental Results}

This section displays the experimental results of our electronic circuit realization on generating a random signal. The response signal at the output of the operational amplifier element is then measured by using a computer digital oscilloscope. Therefore, a signal sample set is registered by varying the potentiometer in the circuit shown in Figure 2. Hence, the voltage generated by this potentiometer at its central pin, and here named as $V_{m}$, is read to label the potentiometer action to each output outcome and presented in Figures 4 to 9. For each one figure, there is the related signal and its histogram. 

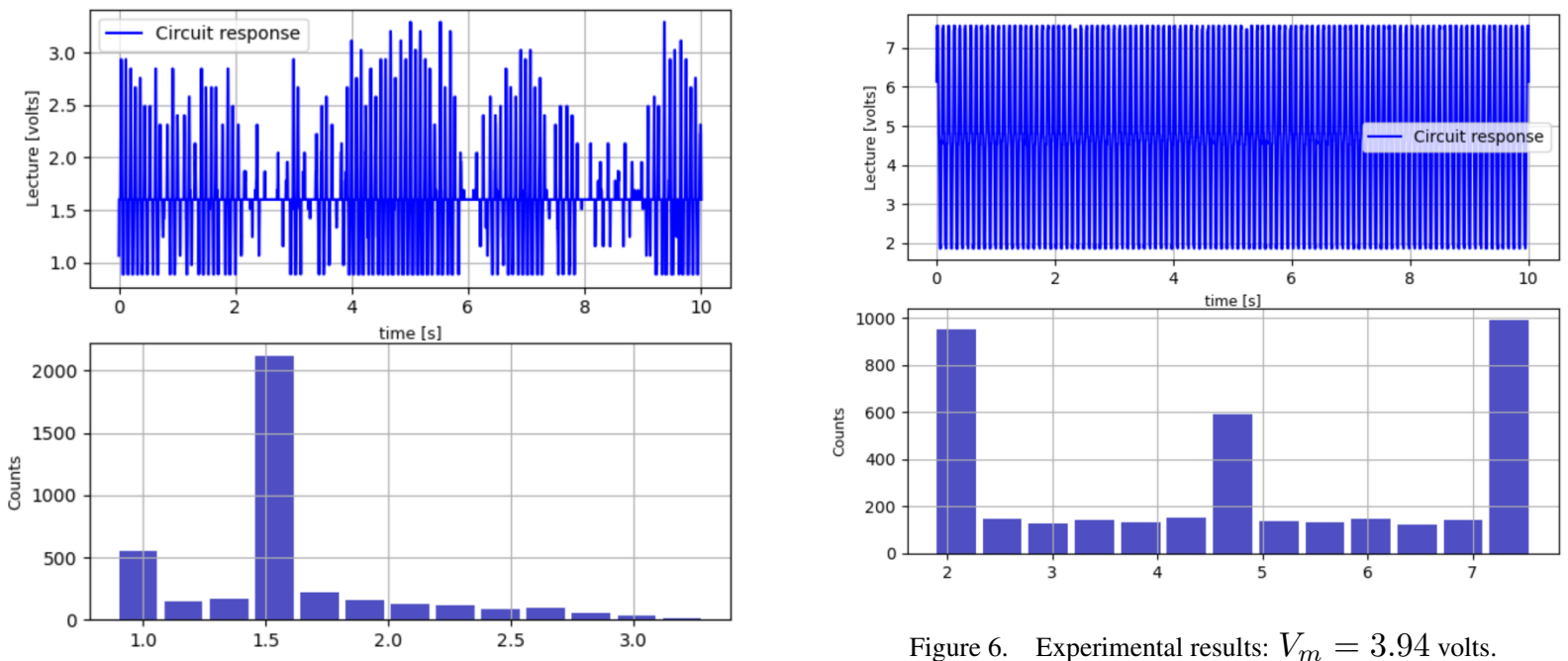

Figure 6. Experimental results: $V_{m}=3.94$ volts.

Figure 4. Experimental results: $V_{m}=0.93$ volts.
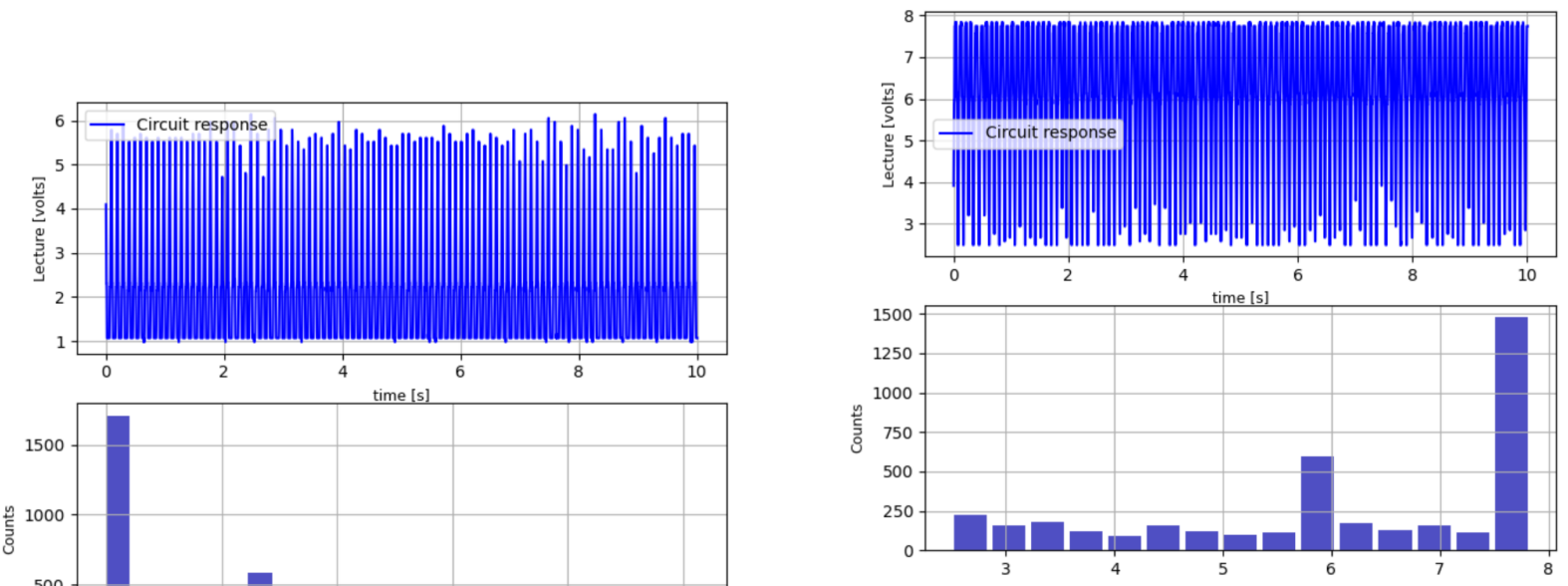

Figure 7. Experimental results: $V_{m}=5.26$ volts.

Figure 5. Experimental results: $V_{m}=1.56$ volts.
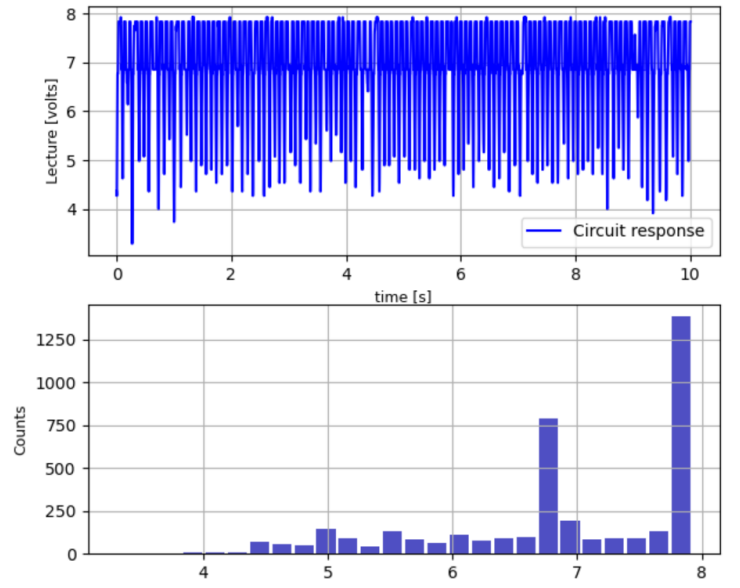

Figure 8. Experimental results: $V_{m}=6.05$ volts. 

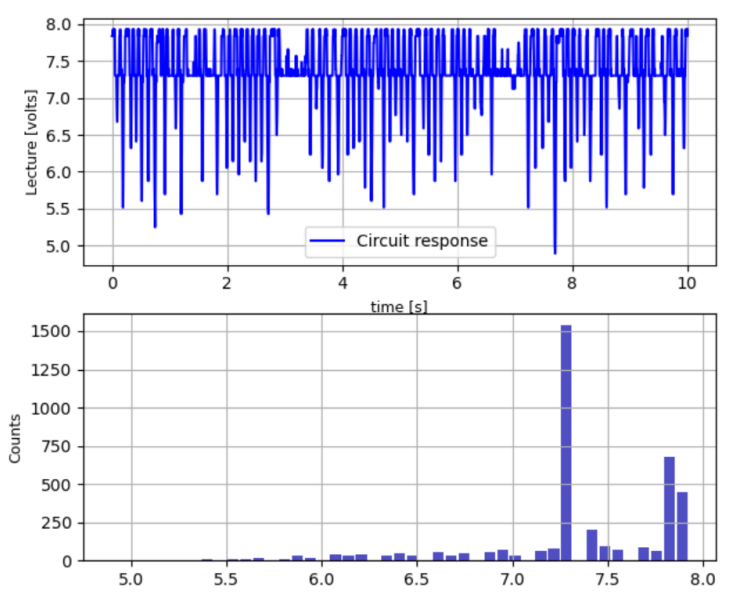

Figure 9. Experimental results: $V_{m}=6.53$ volts.

\section{Conclusion}

A simple and low-cost electronic circuit design to produce a random signal was proposed. Additionally, a potentiometer was included to tune this signal for further reading. To note, by changing the capacitors data linked to the related transistors, other kinds of random signals are possible. This offers more further options on the proposed circuit design. Finally, to highlight that the histogram given, for instance, in Figure 6, resembles the one reported in [Evangelista et al., 2017]. In other words, the generated random signal may be a chaotic one, but we decided not to test it as a chaotic signal. Finally, and related to cybernetic systems, our approach uses feedback to an electronic circuit to produce a self-sustained dynamic response. This may be a cybernetic method.

\section{Acknowledgements}

This research was completely funded by the Spanish Ministry of Economy and Competitiveness (State Research Agency of the Spanish Government)/Fondos Europeos de Desarrollo Regional (MINECO/FEDER), grant number DPI2015-64170-R.

\section{References}

Buchovecká, S., Lórencz, R., Kodỳtek, F., and Buček, J. (2017). True random number generator based on ring oscillator puf circuit. Microprocessors and Microsystems, 53, pp. 33-41.
Evangelista, J. V., Artiles, J. A., Chaves, D. P., and Pimentel, C. (2017). Emitter-coupled pair chaotic generator circuit. AEU-International Journal of Electronics and Communications, 77, pp. 112-117.

Garber, E. and Rozenvasser, E. (1965). The investigation of periodic regimes of nonlinear systems on the basis of the filter hypothesis(periodic regimes of nonlinear systems investigated on basis of filter hypothesis). AUTOMATION AND REMOTE CONTROL, 26, pp. 274-285.

Karakaya, B., Gülten, A., and Frasca, M. (2019). A true random bit generator based on a memristive chaotic circuit: Analysis, design and fpga implementation. Chaos, Solitons \& Fractals, 119, pp. 143-149.

Khalil, H. K. and Grizzle, J. W. (2002). Nonlinear systems, vol. 3. Prentice hall Upper Saddle River, NJ.

Minati, L., Gambuzza, L., Thio, W., Sprott, J., and Frasca, M. (2020). A chaotic circuit based on a physical memristor. Chaos, Solitons \& Fractals, 138, pp. 109990.

Pham, V.-T., Akgul, A., Volos, C., Jafari, S., and Kapitaniak, T. (2017). Dynamics and circuit realization of a no-equilibrium chaotic system with a boostable variable. AEU-International Journal of Electronics and Communications, 78, pp. 134-140.

Piper, J. R. and Sprott, J. C. (2010). Simple autonomous chaotic circuits. IEEE Transactions on Circuits and Systems II: Express Briefs, 57 (9), pp. 730-734.

Slotine, J.-J. E., Li, W., et al. (1991). Applied nonlinear control, vol. 199. Prentice hall Englewood Cliffs, NJ.

Vazquez-Medina, R., Díaz-Méndez, A., del Río-Correa, J., and López-Hernández, J. (2009). Design of chaotic analog noise generators with logistic map and mos qt circuits. Chaos, Solitons \& Fractals, 40 (4), pp. 17791793.

Wu, S. (1987). Chua's circuit family. Proceedings of the IEEE, 75 (8), pp. 1022-1032.

Yildirim, M. and Kacar, F. (2020). Chaotic circuit with ota based memristor on image cryptology. $A E U$ International Journal of Electronics and Communications, 127, pp. 153490.

Zaitceva, I. and Chechurin, L. The estimation of aircraft control system stability boundaries by the describing function method, cybernetics and physics, 2020, vol. 9, no. 2. URL: https://doi. org/10.35470/2226-41162020-9-2-117-122, pp. 117-122.

Zhou, W.-j., Wang, Z.-p., Wu, M.-w., Zheng, W.-h., and Weng, J.-f. (2015). Dynamics analysis and circuit implementation of a new three-dimensional chaotic system. Optik, $126(7-8)$, pp. 765-768. 